\title{
Methods of Topological Obstruction Theory in Condensed Matter Physics
}

\author{
I. A. Ovid'ko ${ }^{1}$ and A. E. Romanov ${ }^{2}$ \\ 1 Physico-Mechanical Department, M. I. Kalinin Polytechnical Institute, SU-195251 Leningrad, \\ USSR \\ ${ }^{2}$ Theoretical Department, A. F. Ioffe Physico-Technical Institute, SU-194021 Leningrad, USSR
}

\begin{abstract}
The notion of relative topological textures - nonuniform states of the general type in condensed (ordered) media - is introduced. For the classification of such states, an effective method is proposed which is based on the topological obstruction theory. The examples of relative topological textures are examined within the framework of the approach studied here.
\end{abstract}

\section{Introduction}

Topological methods are now recognized as being of great importance in condensed matter physics (for a review, see [1-4]) as well as in gauge theories of elementary particles (e.g. [5-8]). The former application of the homotopic topology allows one to classify defects and topological textures in condensed media in such a way that a large (but finite) energy barrier exists for the transformation of a defect (or a topological texture) from one class into another. Such media as ferromagnets, crystals, liquid crystals, superconductors, and superfluids have been effectively examined within the framework of the above approach.

The procedure for the topological classification has its roots in the idea that a condensed (ordered) medium is characterized by the order parameter $f(x)$ which is assumed to be a continuous map $f: K \rightarrow V$ from the physical space $K$ occupied by the medium into the order parameter space $V$ describing the internal symmetry properties of the medium. More precisely, $V$ is a quotient space $G / H$, where $G$ and $H$ are taken to be groups of broken and unbroken symmetry of the medium, respectively. When studying the homotopic classification of maps $K \rightarrow V$ we come to the classification of the order parameter configurations - nonuniform states of the medium. Continuous deformations of the order parameter require a small amount of energy as contrasted to discontinuous ones. Therefore the topological classification of the order parameter configurations seems to be relevant and natural from the energetic point of view. The above statement is well supported by an experimental investigation of defects in ordered media [1-4]. 
At present, however, only a small part of the order parameter configurations (defects, topological textures, cores of defects [9], solitons [10]) is an object of the topological analysis in condensed matter physics. The main aim of the present study is to extend and continue the applications of topological methods to the examination of the general type (but not the most general type, see below) of nonuniform states in ordered media. The paper is organized as follows. Section 2 deals with the general classification algorithm which is known as the topological obstruction theory. In Sect. 3 we are concerned with some special cases of the algorithm. Section 4 is devoted to the application of the topological obstruction theory to the examination of physically interesting examples. In the last section one can find the concluding remarks.

\section{Relative Topological Textures. Elements of the Obstruction Theory}

Consider an ordered medium which occupies a region $K$ in the physical space. Let the order parameter have some continuous and later fixed distribution (values) in the area $M$ of the $K$, i.e. we have a continuous map

$$
f: M \rightarrow V
$$

where $V$ is the order parameter space of the medium. The following question is of great significance: is it possible to extend continuously the map (1) to the whole region $K$, i.e. is it possible to construct a continuous map

$$
g: K \rightarrow V
$$

such that its restriction on $M$ is equal to the map $f$

$$
\left.g\right|_{M}=f ?
$$

In the case of a positive answer to the above question the map (2) will describe some state (the order parameter configuration) of the ordered medium in the region $K$. We shall refer to such states as topological textures relative to $f: M \rightarrow V$ or simply as relative topological textures (RTTs). A brief discussion of the aforementioned states may be also found in [2].

RTTs are the medium states of a rather general type, and by varying $K, M, f$ it is possible to construct a large class of physically interesting states. For example, topological textures correspond to the case when $M$ is a medium boundary and $f: M \rightarrow v$; here $v$ is a point from $V$. Defects also represent a particular case of RTTs; for their analysis we must take $M=0$ and suppose that $K$ is the subregion where the order parameter is a continuous map. But we think RTTs are not the most general type of ordered media states (for details, see Sect. 5).

Let us discuss now the question of RTTs' classification. It appears that in topology there is a branch dealing with the problem of determining and dividing into homotopy classes the set $W$ of all possible continuous maps under the conditions (1)-(3), i.e. under the map $f: M \rightarrow V$ given in advance. This branch is known as the topological obstruction theory [11-13]. The set $W$ is the collection of all topological textures relative to $f: M \rightarrow V$, and the division of $W$ into the homotopy classes is identical to the division of RTTs into the disjoint classes. 
Moreover, one can conclude after some consideration of energetics (continuous deformations of the order parameter cost a small energy, whereas for singular deformations one needs a large energy quantity) that the classes of RTTs set must be thought of as the ones being divided by considerable energy barriers. Below we give briefly the account of the methods of topological obstruction theory, a complete description of which may be found in [11-13].

The initial conditions of the problem are as follows: the sets $K, M(M \subset K)$ and the map $f: M \rightarrow V$ are given. Hereafter we make the simplification by assuming $K$ to have only one connected piece and $V$ to be $d$-simple space, where $d \geqq \operatorname{dim} K$. Let us extend the map $f$ over the entire region $K$. For the purpose of what follows it will be convenient to divide $K$ into $C W$-complex ${ }^{1}$. By $K^{n}$ we mean the $n$-dimensional sceletion of $C W$-complex, i.e. its subcomplex which consists of all cells with the dimension not exceeding $n . \bar{K}^{n}$ will be said to be a subcomplex $K^{n} \cup M$. Further we shall use in our discussion the concept of $n$-homotopy. Let continuous maps $g: K \rightarrow V$ and $s: K \rightarrow V$ be identical on $M$,i.e. $\left.g\right|_{M}=\left.s\right|_{M}$. Then the maps $g$ and $s$ are $n$-homotopic if their restrictions $\left.g\right|_{\bar{K}^{n}}$ and $\left.s\right|_{\bar{K}^{n}}$ on $\bar{K}^{n}$ are homotopic relative to $M$, i.e. there exists a homotopy $h_{t}, 0 \leqq t \leqq 1$ such that

$$
h_{0}=\left.g\right|_{\bar{K}^{n}}, \quad h_{1}=\left.s\right|_{\bar{K}^{n}},\left.\quad h_{t}\right|_{M}=\left.g\right|_{M}=\left.s\right|_{M}
$$

for all $0 \leqq t \leqq 1$. If $M=0$ one can say instead of " $n$-homotopy relative to $M$ " simply "n-homotopy."

Thus, let $f: M \rightarrow V$ be a fixed continuous map and $W$ the set of all possible continuous extensions of the map $f$ over $K$,

$$
W=\left\{g: K \rightarrow V \text {, such that }\left.g\right|_{M}=f\right\} .
$$

We would like to recall that this set $W$ is just the set of all topological textures relative to $f: M \rightarrow V$. In order to divide the set $W$ into homotopy classes (in other words to classify RTTs) one must characterize these classes by some quantities.

The relation of $n$-homotopy relative to $M$ divides the set $W$ into the disjoint $n$-homotopy classes relative to $M$. For any $n \geqq 1$ every $(n-1)$-homotopy class contains in general a collection of $n$-homotopy classes relative to $M$. Then the classification problem will be solved if we enumerate all the $n$-homotopy classes contained in a given $(n-1)$-homotopy class with the aid of some homology or cohomology invariants.

Such an enumeration appears to be possible. In order to receive it, some additional auxiliary mathematical notions should be used. Consider the group $R^{n}(K, M ; b)$ whose elements $\alpha$ are the classes of such homotopies $h_{t}: \bar{K}^{n-1} \rightarrow V$, $0 \leqq t \leqq 1$ that

$$
h_{0}=\left.b\right|_{\bar{K}^{n-1}}=h_{1},\left.\quad h_{t}\right|_{M}=\left.b\right|_{M}, \quad 0 \leqq t \leqq 1,
$$

where by $b$ we mean an element of the set $\Omega$ of all continuous maps $a: \bar{K}^{n-1} \rightarrow V$ such that $\left.a\right|_{M}=\left.b\right|_{M}$. In other words

$$
R^{n}(K, M ; b)=\pi_{1}\left(\Omega, \omega_{0}\right),
$$

${ }^{1}$ All mathematical concepts and terms used may be found in [11-13] 
where $\omega_{0}=\left.b\right|_{\bar{K}^{n-1}}$. Every homotopy $h_{t}: \bar{K}^{n-1} \rightarrow V, 0 \leqq t \leqq 1$ satisfying (6), determines the cohomology class $\delta^{n}\left(b, b ; h_{t}\right)$ from the cohomology group $H^{n}\left(K, M ; \pi_{n}(V)\right)$ with coefficients in the $n$-dimensional homotopy group $\pi_{n}(V)$.

Designate by $\theta$ some arbitrary, but further on a fixed $(n-1)$-homotopy class. Consider a map $p: K \rightarrow V$ from this class $\theta$. Every map $g \in \theta$ determines the characteristic element $\psi^{n}(p, g) \in Q_{\theta}^{n}\left(K, M ; \pi_{n}(V)\right)$, where

$$
Q_{\theta}^{n}\left(K, M ; \pi_{n}(V)\right)=H^{n}\left(K, M ; \pi_{n}(V)\right) / J_{\theta}^{n}\left(K, M ; \pi_{n}(V)\right)
$$

is the quotient of the cohomology group $H^{n}\left(K, M ; \pi_{n}(V)\right)$ to its subgroup $J_{\theta}^{n}$. Here $J_{\theta}^{n}$ is the image of the group $R^{n}(K, M ; p)$ under the homomorphism

$$
\xi_{n}: R^{n}(K, M ; p) \rightarrow H^{n}\left(K, M ; \pi_{n}(V)\right),
$$

which is determined by the formula

$$
\xi_{n}(\alpha)=\delta^{n}\left(p, p ; h_{t}\right) .
$$

Finally consider one more definition. An element $\beta$ of the group $Q_{\theta}^{n}\left(K, M ; \pi_{n}(V)\right)$ is called $p$-admissible, if there is a map $g \in \theta$, such that $\psi^{n}(p, g)=\beta$.

Taking into account the above mentioned mathematical terms and notions, one can proceed to the study of a classification theorem [12].

Theorem. "Given an (n-1)-homotopy class $\theta$ relative to $M$ of the maps $W$, the $n$-homotopy classes relative to $M$ of $W$ which are contained in $\theta$ are in one-to-one correspondence with the elements of the p-admissible set $A_{p}^{n}$ in $Q_{\theta}^{n}\left(K, M ; \pi_{n}(V)\right)$, where $p$ is an arbitrarily given map in $\theta . "$

Summing up, the classification of RTTs may be reduced to the determination of the sets of $A_{p}^{n}$ type. Direct computation of these sets depends on the concrete conditions of the problem under consideration, i.e. on the structure of sets $K, M, V$, and a map $f$.

\section{Special Cases of a Classification Scheme}

Now we proceed to consider some special cases of a classification scheme. In doing so we shall formulate the conditions which $K, M, V$, and $f: M \rightarrow V$ happen to satisfy in every case and give the suitable algorithms for the computation of the set $D_{W}$ of the homotopic classes of maps from $W$.

1) $M=0, K$ is a 3-dimensional region having a complicated form. The order parameter space $V$ is taken to be a space for which either the fundamental group $\pi_{1}(V)$ or the second homotopic group $\pi_{2}(V)$ is trivial.

Then, according to the obstruction theory, the set of the homotopic classes of the maps $K \rightarrow V$,

$$
D_{W}=H^{1}\left(K ; \pi_{1}(V)\right)
$$

if the group $\pi_{1}(V)$ is nontrivial, and

$$
D_{W}=H^{2}\left(K ; \pi_{2}(V)\right)
$$


if the group $\pi_{2}(V)$ is nontrivial. Here, by $H^{m}(K ; E)$ we mean the $m$-dimensional cohomology group with coefficients in the Abelian group $E^{2}$. This case 1) has also been briefly discussed in $[2,14]$.

The classification procedure based on the formulae (11), (12) is applicable for a superconductor, a ferromagnetic-easy plane, a superfluid ${ }^{4} \mathrm{He}$ (the order parameter space $\left.V=S^{1}\right)$, an isotropic ferromagnetic $\left(V=S^{2}\right)$, a multisublattice antiferromagnetic $(V=S O(3))$. If $V=V_{1} \times V_{2}$, the set of the homotopic classes of the maps $K \rightarrow V$,

$$
D_{W}=D_{W_{1}} \times D_{W_{2}}
$$

where $D_{W_{1}}$ and $D_{W_{2}}$ are taken to be the set of the homotopic classes of the maps $K \rightarrow V_{1}$ and that of the maps $K \rightarrow V_{2}$, respectively. This provides a topological analysis of a superfluid ${ }^{3} \mathrm{He}$ by means of (11), (12).

2) Whereby (11) are classified, also the maps $K \rightarrow V$, with the proviso only that $M=0$ and $K$ is homotopically equivalent to some 1-dimensional space. The question of whether one of the groups $\pi_{1}(V), \pi_{2}(V)$ is trivial, is ignored here. For example, it may be of interest for the classification of nonuniform states in the nematic ( $V=R P_{2}$-projective plane). The case in question is a particular one of the next case.

3) Let us now discuss one more special case of the application of the obstruction theory. The conditions with which we shall be concerned in the below consideration require that $K$ and $M$ are homotopically equivalent to some 1-dimensional or 0 -dimensional spaces $K^{\prime}$ and $M^{\prime}$, respectively. In addition the map $f: M \rightarrow V$ must be homotopic to the constant map (map into a point). In this event the set of classes of topological textures relative to $f: M \rightarrow V$,

$$
D_{W}=H^{1}\left(K, M ; \pi_{1}(V)\right),
$$

where by $H^{1}\left(K, M ; \pi_{1}(V)\right)$ is meant a relative 1-dimensional cohomology group. For illustration purposes in the following section we shall examine some examples of RTTs by the methods discussed above.

\section{Relative Topological Textures} in Superconductors, Liquid Crystals, Ferromagnetics, and Superfluid ${ }^{4} \mathrm{He}$

A) To start with, consider superconductors of a nontrivial form. The order parameter space $V=S^{1}$ of a superconductor is known to be identical to that of a ferromagnetic-easy plane as well as of a superfluid ${ }^{4} \mathrm{He}$. Therefore the following analysis is valid for these systems, too.

Let a superconducting sample have the form of a filled sphere with $r$ handles $N_{r}$ (Fig. 1) (filled torus is $N_{1}$ ). According to the formula (11) the maps $N_{r} \rightarrow S^{1}$ are classified by the elements of the cohomology group,

$$
H^{1}\left(N_{r} ; \pi_{1}\left(S^{1}\right)\right)=\underbrace{Z \times \ldots \times Z}_{r} .
$$

\footnotetext{
${ }^{2}$ Systematical presentation of a cohomology theory may be found in the books $[11,13]$
} 


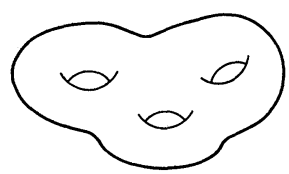

Fig. 1. The filled sphere with $r$ handles, $r=3$

Consequently, states of the superconductor having the $N_{r}$-form are classified by the sets $\left(z_{1}, \ldots, z_{r}\right)$, where $z_{1}, \ldots, z_{r}$ are integers.

Let a superconducting sample have now $T_{r}$-form, where $T_{r}$ is a 3-dimensional region which is homotopically equivalent to a 2-dimensional sphere with $r$ handles. Using the formula (11) one can easily verify that the maps $T_{r} \rightarrow S^{1}$ are classified by the elements of the group

$$
H^{1}\left(T_{r} ; \pi_{1}\left(S^{1}\right)\right)=\underbrace{Z \times \ldots \times Z}_{2 r} .
$$

Hence, possible non-uniform states of the $T_{r}$-superconductor are characterized by the sets $\left(z_{1}, \ldots, z_{2 r}\right)$ of integers.

One can extend these results to the classification of nonuniform states in a multisublattice antiferromagnetic of $N_{r}$-form ( $T_{r}$-form, respectively) due to the replacement of the group $Z$ in the formula (15) [formula (16), respectively] by the two-element group

$$
\pi_{1}(S O(3))=Z_{2}=(1,-1) .
$$

The same replacement, if the analysis of the $N_{r}$-form nematic is desired, takes place (see the case 2 of the previous section, because $N_{r}$ is homotopically equivalent to an one-point union of circles, which is a 1-dimensional space).

B) Consider an isotropic ferromagnetic having $C_{r}$-form, where $C_{r}$ is a cube with $r$ internal pores. The homotopic classes of the maps $C_{r} \rightarrow S^{2}$ are in one-to-one correspondence with the elements of the group

$$
H^{2}\left(C_{r} ; \pi_{2}\left(S^{2}\right)\right)=\underbrace{Z \times \ldots \times Z}_{r} .
$$

Thus, states of the $C_{r}$-form isotropic ferromagnetic are classified by the sets $\left(z_{1}, \ldots, z_{r}\right)$ of integers.

C) For illustration of case 3 we shall study now the nematic $\left(V=R P_{2}\right)$ placed between two parallel plane surfaces. If the surfaces affect (fix) the orientation of the nematic molecules near them, the accounting of the fixed map $f: M \rightarrow R P_{2}$, becomes relevant. Here $M$ is the union of the aforementioned surfaces. We may think of the molecule orientation as being perpendicular to the surfaces on $M$. Then the $f$ maps $M$ into a point of $R P_{2}$.

Examine the topological classification of line defects, which are parallel to the surfaces (Fig. 2). In this event the regions $K$ and $M$ are homotopically equivalent to a circle $S^{1}$ and a 0 -dimensional sphere (two points) $S^{0}$, respectively. 


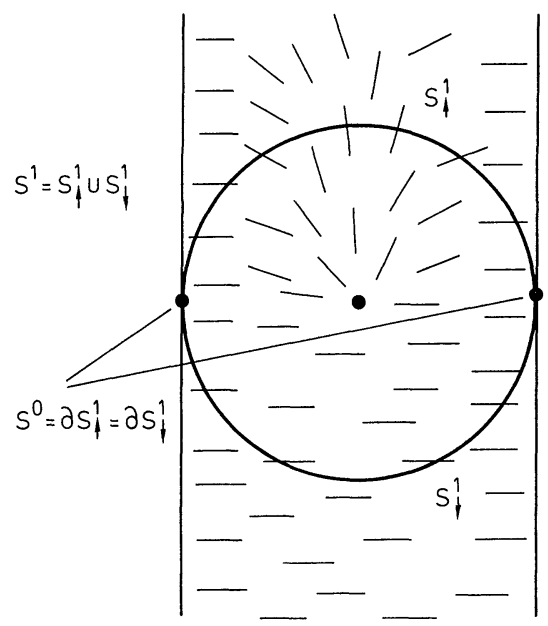

Fig. 2. The nematic liquid crystal placed between two parallel surfaces. The orientation of the nematic molecules is strictly fixed near surfaces (the molecules are perpendicular to the surfaces). The topologically stable line defect, surrounded by the circle $S^{1}=S_{\uparrow}^{1} \cup S_{\downarrow}^{1}$, is pictured. $S^{0}=\partial S_{\uparrow}^{1}=\partial S_{\downarrow}^{1}$

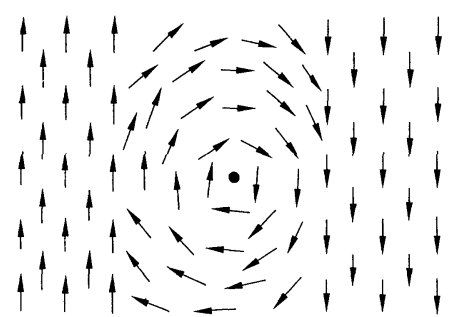

Fig. 3. The vortex within a domain wall core a ferromagnetic-easy axis

Bearing in mind the formula (14) we conclude that the maps $K \rightarrow R P_{2}$ are classified by the sets $(j, l)$ - the elements of the group,

$$
H^{1}\left(K, M ; \pi_{1}\left(R P_{2}\right)\right)=H^{1}\left(S^{1}, S^{0} ; Z_{2}\right)=Z_{2} \times Z_{2} .
$$

In doing so $j$ ( $l$, respectively) characterizes the homotopic class of the map $S_{\uparrow}^{1} \rightarrow R P_{2}$ $\left(S_{\downarrow}^{1} \rightarrow R P_{2}\right.$, respectively) under the fixed map $S^{0}=\partial S_{\uparrow}^{1}=\partial S_{\downarrow}^{1} \rightarrow R P_{2}$ (Fig. 2). The symmetric sets $(1,1)$ and $(-1,-1)$ describes unstable defects because the molecule orientation field along $S_{\uparrow}^{1}$ can be continuously deformed to the field along $S_{\downarrow}^{1}$. On the contrary, any defect with asymmetric set $[(1,-1)$ or $(-1,1)]$, is topologically stable due to the distinction between the homotopic classes of the maps $S_{\uparrow}^{1} \rightarrow R P_{2}$ and $S_{\downarrow}^{1} \rightarrow R P_{2}$. Such a relation between the defects stability and the sets symmetry has a general character. We shall see this for different physical situations.

D) Let us consider from a topological point of view the vortices in a domain wall of a ferromagnetic-easy axis (Fig. 3). Within the core of the wall the order parameter space $V=S^{1}$. Outside the core (in domains) the order parameter - a magnetization vector $M$ is fixed. For instance, the situation when on the left (on the 
right) of the core the vector $M$ is strictly directed upwards (downwards), is depicted in Fig. 3. In this event the spaces $K$ and $M$ are homotopically equivalent to $S^{1}$ and $S^{0}$, respectively. As a corollary, for the classification of the vortices we can use the formula (14)

$$
D_{W}=H^{1}\left(K, M ; \pi_{1}\left(S^{1}\right)\right)=H^{1}\left(S^{1}, S^{0} ; Z\right)=Z \times Z .
$$

Topological classes of vortices are characterized by the sets $\left(z_{1}, z_{2}\right)$ of integers. The physical interpretation of (19) turns out to be similar with the previous case C): the symmetric sets correspond to the topologically unstable defects whereas the asymmetric sets characterize the topologically stable defects.

Next, we shall briefly study some RTTs not falling under the special cases discussed in Sect. 3.

E) There may be present point defects in the nematic placed in the cylinder, near the lateral surface of which the nematic molecules have a fixed orientation [for example, normally to the cylinder lateral surface (Fig. 4)] [15]. Such defects are classified by the sets $\left(z_{1}, z_{2}\right)$ - the elements of the group

$$
H^{2}\left(S^{2}, S^{1} ; \pi_{2}\left(R P_{2}\right)\right)=Z \times Z .
$$

The topologically stable defects are characterized by the asymmetric sets $\left(z_{1}, z_{2}\right)$, $z_{1} \neq z_{2}$ the topologically unstable defects by the symmetric sets $\left(z_{1}, z_{2}\right), z_{1}=z_{2}$.

If a magnetic field $\mathbf{H}$ (exceeding some threshold value) appears then the Frederix transition in the nematic system emerges, i.e. the nematic molecules tend to be oriented parallel with $\mathbf{H}$. Let the magnetic field be parallel with the cylinder axis. Then the nematic molecules in the central part of the cylinder are strictly cooriented with $\mathbf{H}$, whereas the molecules near the cylinder surface persist to be perpendicular to the surface. As a corollary, there is a region (between the central part and the lateral surface of the cylinder) in which the molecules do not have a fixed orientation. The classification of the order parameter configurations in the above region is an exact copy of the classification in the double cylinder shown in Fig. 5.

Apply the methods of the obstruction theory to the examination of a line loop defect once surrounding the internal cylinder. By doing so we come to the conclusion that the classes of such defects are in one-to-one correspondence with

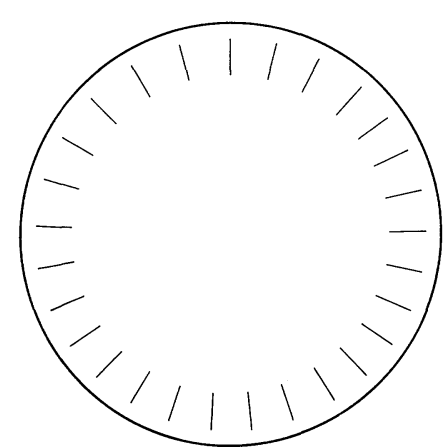

Fig. 4. The section of a cylinder vessel with a nematic liquid crystal. A fixed distribution of the nematic molecules near the vessel surface is shown 


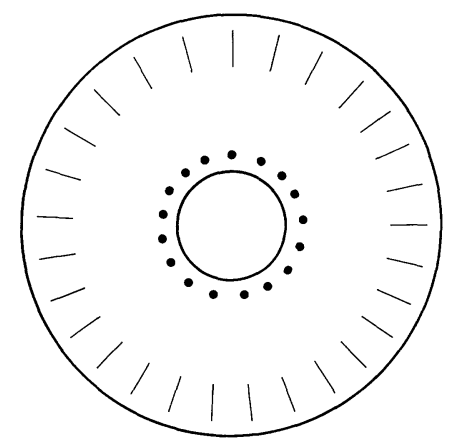

Fig. 5. The section of a double cylinder vessel with a nematic liquid crystal. A fixed distribution of the nematic molecules near the vessel surface is shown

the elements of the group

$$
H^{1}\left(S^{1}, S^{0} ; \pi_{1}\left(R P_{2}\right)\right) \times H^{2}\left(S^{2}, S^{1} ; \pi_{2}\left(R P_{2}\right)\right)=Z_{2} \times Z_{2} \times Z \times Z .
$$

The symmetry of every characteristic set $\left(j, l, z_{1}, z_{2}\right)$ indicates stability properties of the defect: the case $j=l, z_{1}=z_{2}$ reflects the unstability of the defect, whereas any other combination of $j, l, z_{1}, z_{2}$ labels the topologically stable defect. Here $j, l \in Z_{2}$; $z_{1}, z_{2} \in Z$.

F) Let us consider topological texture with $r$ point defects in an isotropic ferromagnetic $\left(V=S^{2}\right)$. For co-existence of the texture and the defects, the common topological charge of these defects must be zero. In other words the map of any 2-dimensional sphere, surrounding all defects, is homotopic to the constant map (map into a point of the order parameter space). One can prove after the use of the obstruction theory methods that topological textures with $r$ point defects $(r \geqq 2)$ are classified by the elements of the group

$$
\pi_{3}\left(S^{2}\right) \times H^{2}\left(B_{r-1} ; \pi_{2}\left(S^{2}\right)\right)=\underbrace{Z \times \ldots \times Z}_{r},
$$

where by $B_{r-1}$ we denote the one-point union of $(r-1)$ spheres.

\section{Concluding Remarks}

As shown in foregoing sections of the present paper the obstruction theory is an effective tool for the investigation of nonuniform states in condensed media. By the use of this theory one can study the two important problems:

1. The analysis of states in media occupying volumes of a complicated form.

2. The analysis of states under a fixed distribution of the order parameter in some part of a volume occupied by medium.

In addition one should consider the obstruction theory to be a natural starting point for the topological description of the most general type of media states. We shall discuss it after the next introductory paragraph. 
There exists physical interest in nonuniform states, for which in some region $\tilde{M}$ of a volume $K$ occupied by a medium, the order parameter can have its value only in some subsurface $\widetilde{V}$ of the order parameter space $V$. In other words for such a state description, one has to examine extensions of an unfixed ${ }^{3}$ map $\tilde{f}: \tilde{M} \rightarrow \tilde{V}$ to the map $g: K \rightarrow V$, where $\tilde{M} \subset K, \tilde{V} \subset V$. Namely this kind of the order parameter configurations includes in itself cores of defects [9] and solitons [10].

Now we have all the ingredients to construct the most general type of media states. Generally speaking a state of this type is characterized by the map $g: K \rightarrow V$ whose restrictions on $M$ and $\tilde{M}(M \subset K, \tilde{M} \subset K, M \cap \tilde{M}=0)$ are taken to be a fixed map $f: M \rightarrow V$ and an unfixed map $\tilde{f}: \tilde{M} \rightarrow \tilde{V}(\tilde{V} \subset V)$, respectively. As far as we know, the homotopic classification of the maps fails now to be created. But it is hard to see how the basis of this classification could be anything other than the obstruction theory.

It is of interest to note that when considering the classification of gauge field singularities (à la Dirac monopole), the obstruction theory is also relevant. Indeed, within the geometrical interpretation of gauge theories (fiber bundle formalism [5-8]) the classification of gauge field singularities is nothing but the principal bundle classification which can be reduced to the homotopic one of the maps $X \backslash Y \rightarrow B_{G}$ [16-18]. Here, gauge field is singular on the region $Y$ of Minkowski space $X$ (in other words $X \backslash Y$ is the fiber bundle base), and by $B_{G}$ we mean the base of universal fiber bundle corresponding to the principal $G$-bundle which is a geometrical analogue of the gauge theory in question. Thus we again come face to face with the homotopic classification of maps and, as a corollary, with the obstruction theory.

It should be noted that the discussed approach cannot be applied without modifications to studies of nonsingular excitations (à la Nielsen-Olesen tube, t'Hooft-Polyakov monopole, etc.) in gauge fields. Detailed consideration of topological problems taking place in gauge theories is beyond the scope of the present paper, but we want to emphasize that there are many common points of the applications of topology to condensed matter physics and to gauge theories. The use of the obstruction theory is only one such point.

Acknowledgements. Many thanks are due to Prof. V. I. Vladimirov and Dr. S. A. Popov for helpful discussions.

\section{References}

1. Mermin, N.D.: The topological theory of defects in ordered media. Rev. Mod. Phys. 51, 591-648 (1979)

2. Michel, L.: Symmetry defects and broken symmetry. Configurations. Hidden symmetry. Rev. Mod. Phys. 52, 617-651 (1980)

3. Mineev, V.P.: Topologically stable inhomogeneous states in ordered media. Sov. Sci. Rev. A2, 173-234 (1980)

4. Trebin, H.-R.: The topology of non-uniform media in condensed matter physics. Adv. Phys. 31, 195-254 (1982)

5. Jaffe, A., Taubes, C.: Vortices and monopoles. Boston, Basel, Stuttgart: Birkhäuser 1980

${ }^{3}$ We would like to recall that in studying RTTs we deal with a fixed map $f: M \rightarrow V$ 
6. Leinaas, J.M.: Topological charges in gauge theories. Fortschr. Phys. 28, 579-631 (1980)

7. Konopleva, N.P., Popov, V.N.: Gauge fields. New York: Harwood 1981

8. Geometric techniques in gauge theories. Lecture Notes in Mathematics, Vol. 926. Berlin, Heidelberg, New York: Springer 1982

9. Mermin, N.D., Mineyev, V.P., Volovik, G.E.: Topological analysis of the cores of singularities in ${ }^{3} \mathrm{He}-\mathrm{A}$. J. Low Temp. Phys. 33, 117-126 (1978)

10. Mineyev, V.P., Volovik, G.E.: Planar and linear solitons in superfluid ${ }^{3}$ He. Phys. Rev. B 18, 3197-3203 (1978)

11. Spanier, E.H.: Algebraic topology. New York, San Francisco, St. Louis, Toronto, London, Sydney: McGraw-Hill 1966

12. Hu, S.-T.: Homotopy theory. New York, London: Academic Press 1959

13. Dubrovin, B.A., Novikov, S.P., Fomenko, A.T.: Modern geometry: Methods of homology theory. Moscow: Nauka 1984 (in Russian)

14. Kleman, M., Michel, L.: Spontaneous breaking of euclidean invariance and classification of topology stable defects and configurations of crystals and liquid crystals. Phys. Rev. Lett. 40, 1387-1390 (1978)

15. Physics of defects, Balian, R., Kleman, M., Poireir, J.-P. (eds.): Amsterdam, New York, Oxford: North-Holland 1981

16. Husemoller, D.: Fibre bundles. New York, St. Louis, San Francisco, Toronto, London, Sydney: McGraw-Hill 1966

17. Milnor, J.W., Stasheff, J.D.: Characteristic classes. Princeton, NJ : Princeton University Press and University of Tokyo Press 1974

18. Michenko, A.S.: Vector bundles and their applications. Moscow: Nauka 1984 (in Russian)

Communicated by J. Fröhlich

Received September 9, 1985; in revised form December 20, 1985 
